\section{De la evidencia al desempeño: cómo fijar prioridades y tomar buenas decisiones 1}

Franklin White ${ }^{2}$

1 Es traducción de un documento titulado "From evidence to performance: setting priorities and making good decisions", cuyo contenido se presentó durante la sesión plenaria del Simposio sobre Estrategias Nacionales para la Renovación de la Meta de Salud para Todos, Washington, DC, 19 a 20 de febrero de 1998. A pedido del autor, se ha traducido evidence como "evidencia", con el significado exacto del inglés, por ser la acepción actualmente en uso en este campo.

2 Organización Panamericana de la Salud, División de Prevención y Control de Enfermedades, Washington, DC, EUA.
En esta época de respeto por la ciencia médica y por la responsabilidad gerencial, las organizaciones de salud deben rendir cuentas por sus acciones con creciente frecuencia. La época en que se determinaban las prioridades y se tomaban las decisiones sobre bases políticas o acatando el consejo intuitivo de asesores respetados y bien intencionados ha dado paso gradualmente a una era que valora la demostración de efectividad y eficiencia. Las decisiones relativas a las prioridades y a los programas deben basarse firmemente en conocimientos comprobados y someterse a evaluación continua. Esta transición requiere promover el pensamiento crítico en todos los ámbitos. Surge entonces la siguiente pregunta: ¿Cómo podemos promover el razonamiento crítico en los servicios de salud para fijar prioridades, adoptar decisiones acertadas y poner en práctica iniciativas efectivas? En otras palabras, ¿cómo podemos pasar de la evidencia al desempeño? Una pregunta aún más básica es ¿por qué debemos fijar prioridades?

\section{¿POR QUÉ SE ESTABLECEN PRIORIDADES?}

La respuesta más fundamental a la pregunta aquí planteada es que siempre hemos establecido prioridades. Las prioridades fijadas en épocas pasadas no eran el resultado de análisis sistemáticos basados en criterios formales, pero el hecho mismo de que se tomaran decisiones implica que se ponderaban varias consideraciones. En algunas sociedades coloniales se creía que toda comunidad de relieve debía tener un hospital. Independientemente de que ello permitiera o no atender a una necesidad acuciante de salud, una comunidad donde hubiera hospital tenía mayor prestigio que otra que no lo tuviera. Era una buena estrategia política y favorecía la actividad comercial en la localidad. En épocas pasadas la fijación de prioridades era un proceso implícito.

Más allá de la política y la tradición, existen otras razones de carácter científico y administrativo para fijar prioridades.

- Las necesidades de salud cambian. Todos los países han pasado por transiciones demográficas y epidemiológicas. En 1985, la razón de enfermedades no transmisibles a transmisibles en lo referente a mortalidad era de aproximadamente 1,5:1 en América Latina y el Caribe. Sin embargo, en el 
año 2000 esta razón se acercará a 3,4:1 y en 2015, a 7:1 (1). Se observan tendencias similares en cuanto a la frecuencia de discapacidad. En definitiva, las prioridades, decisiones y sistemas de atención de salud establecidos hace 20 años, o hace apenas 10, no son los adecuados para hacer frente a tan grandes incrementos de la carga de morbilidad.

- Los conceptos de salud varían. El término tan ampliamente utilizado en las Américas de "enfermedad degenerativa crónica" es ejemplo de una expresión desactualizada, puesto que implica una irreversibilidad que no concuerda con los nuevos conocimientos adquiridos. En presencia de determinadas actitudes socioculturales, es indispensable combatir los mitos con hechos. Antes de que se elaborara la vacuna antisarampionosa prevalecía una actitud nihilista similar con respecto al sarampión, que se consideraba una infección inocua de la infancia y parte natural del crecimiento. Ahora se sabe que la mayoría de las enfermedades crónicas son prevenibles o reversibles y que en muchos casos su detección temprana previene la aparición de complicaciones. Según Peter Drucker, destacado experto en el campo de la gerencia, vale más tomar medidas acertadas que hacer bien las mismas cosas que se han hecho siempre.

- Siempre hay más necesidades de salud que recursos para satisfacerlas. En un sentido implícito, siempre ha habido un proceso de razonamiento. He aquí los interrogantes básicos: ¿"Cuáles" son las necesidades y los recursos? ¿"A quién" pertenecen? ¿"Cuándo", "dónde" y "por qué" existen estas necesidades? ¿Cómo deben tomarse las decisiones y quién debe hacerlo? Por ende, la fijación de prioridades es una cuestión de ética, aun más en los países pobres donde solo una pequeña minoría de las personas que tienen insuficiencia renal, por poner un ejemplo, tiene acceso a servicios de diálisis.

- Los recursos de salud son patrimonio de la sociedad. Independientemente del volumen de recursos de salud que pertenezca al sector privado, desde que el financiamiento público se hizo indispensable para atender a las necesidades de salud, su uso ha reflejado en el terreno práctico los valores sociales y culturales. En su reciente visita a Cuba, el Papa afirmó elocuentemente que "las fuerzas ciegas del mercado" no reflejan debidamente los valores sociales. El recién celebrado Foro Canadiense sobre la Salud ha colocado los valores sociales en el núcleo del proceso; solo después de definir esos fundamentos se permitió que los instrumentos cuantitativos y cualitativos guiaran el camino (2).
- Las buenas prácticas administrativas exigen la asignación racional de los recursos. En otra época, la atención de salud era una empresa relativamente ineficaz pero humanitaria; hoy en día se ha convertido en una disciplina basada en la acumulación de evidencia y sensible a los fenómenos económicos. Actualmente la dificultad radica en determinar cómo se puede lograr que esto se cumpla en el contexto verdadero de sistemas de salud que han surgido como resultado de una combinación de influencias políticas y tradiciones.

\section{¿CÓMO SE FIJAN LAS PRIORIDADES EN LA REALIDAD?}

Para los profesionales de salud con formación científica, es difícil pasar del mundo de lo exacto al del "más o menos". Las autoridades normativas y decisorias en el fondo no están interesadas en la exactitud. Les basta con hacer estimaciones "aproximadas": desean saber si el problema es pequeño o grande, si empeora o mejora, cuánto tiempo tardará en resolverse (a menudo están pensando en los ciclos electorales) y qué puede hacerse al respecto. En sus decisiones entran en juego, además de insumos técnicos, diversas circunstancias y oportunidades, la influencia de los grupos de presión y algunas limitaciones psicosociales (o "momentos de debilidad"). Suele haber más interés en la factibilidad económica de una opción que en sus consecuencias económicas (ya que es otro, quizá la descendencia, quien se deberá preocupar por ellas).

Hay una lucha entre valores conflictivos, como entre la búsqueda de equidad y la facultad de libre elección. Ninguna sociedad puede tenerlo todo. Los valores están arraigados, son estables y son característicos de individuos y grupos. En cambio, las opiniones son pasajeras, entrañan "concesiones recíprocas", pueden formularse sin mucha reflexión y pueden cambiar de un día para otro. La opinión pública en materia de salud hace que la elección libre sea algo más que una mera cuestión de políticas y que se convierta en un asunto político.

El movimiento que se propone dar mayor relieve a la función de la evidencia promete mejorar la calidad del proceso de fijar prioridades y tomar decisiones, que abarca desde las políticas públicas hasta la atención médica individual. Sin embargo, existen también ciertas verdades esenciales que los defensores de intereses deben enfrentar.

- Aun cuando se dispone de evidencia fehaciente, siempre hay otras consideraciones. Por ejemplo, la evidencia que vinculan al tabaco con un proceso patológico han sido fehacientes por varios decenios y desde 
1964 sirvieron de base para una política nacional de salud en los Estados Unidos, al igual que en muchos otros países. No obstante, en el transcurso del último año la industria al fin ha admitido esa evidencia y ha tenido que enfrentar cargos de responsabilidad en los tribunales de justicia.

- Las decisiones suelen adoptarse sobre la base de evidencia incompleta. La toma de decisiones constituye una forma de manejar riesgos. Este proceso tiene más de arte que de ciencia, puesto que se trata menos de las probabilidades de tener o no razón (el aspecto científico), que en las repercusiones de tenerla o no tenerla (el aspecto humano). Las instancias decisorias necesitan tener más información para obrar que para publicar, motivo por el cual los investigadores nunca ejercerán la influencia que les gustaría ejercer en la toma de decisiones. Muy a menudo sucede que, cuando sus resultados "exactos" han pasado por todo un proceso exhaustivo de revisión por pares, el momento para tomar las decisiones ya ha pasado hace mucho tiempo.

- La aplicación de la mejor evidencia disponible no garantiza que las decisiones o los resultados sean acertados, pero mejora la probabilidad de que así sea. Esto es asunto de fe para quienes se encargan de producir la información. Pero no basta con dotar a los individuos de los conocimientos prácticos para que puedan presentar la evidencia (epidemiólogos, economistas, sociólogos y planificadores): los propios encargados de tomar las decisiones deben entender las reglas básicas en torno a cómo se usa la evidencia y mostrarse dispuestos a aplicarla. Las instancias decisorias deben aprender a tomar mejores decisiones.

\section{EL ENFOQUE BASADO EN LA EVIDENCIA}

El enfoque basado en la generación de evidencia consta de cuatro fases interrelacionadas. La primera es el análisis de la situación, que debe consistir no solo en el ejercicio epidemiológico de definir la carga de morbilidad, sino también en describir las características de los servicios de salud en cuanto a diseño y capacidad. La segunda es la evaluación de la eficacia y la efectividad. La evaluación de la eficacia exige prestar atención a las publicaciones sobre intervenciones a fin de determinar si algo "puede funcionar", al menos en circunstancias ideales. Sin embargo, cuando se trata de evaluar la efectividad, el enfoque cambia y la pregunta se convierte en si algo "funciona (o funcionó)" en condiciones reales, interrogante que se relaciona más con las barreras que impiden que la ejecución sea eficaz. Un ejemplo es el examen para la detección del cán- cer cervicouterino, que ha permitido reducir en $70 \%$ la incidencia de cáncer invasor en el Canadá, pero casi imperceptiblemente en la mayoría de los demás países de las Américas (3). Aunque casi todos los países carecen de los recursos necesarios para afrontar directamente los problemas de eficacia, todos pueden examinar la efectividad. La sapiencia adquirida con la experiencia, por medio de actividades sistemáticas de planificación y evaluación y partiendo en ocasiones de proyectos de demostración, constituye el camino que conduce a la mejora de la calidad.

La efectividad y eficiencia de los servicios de salud fueron promovidas a comienzos del decenio de 1970 por el fallecido Archie Cochrane, que empleó la metáfora del encargado de un horno crematorio cuya satisfacción laboral derivaba de observar cuánto entraba y cuán poco salía. Según su propia afirmación, algo similar le sucedía al Servicio Nacional de Salud Británico (4). Sus primeras actividades se reflejan hoy en día en la Colaboración de Cochrane, red de centros dedicados a evaluar las pruebas de eficacia y efectividad.

La tercera fase, que es la de evaluación económica, en principio podría incluir análisis formales de costo-beneficio para ayudar a definir, en términos comparativos, los rendimientos a la inversión producidos por distintas opciones (5). El análisis de costo-eficacia tiene más valor práctico cuando se elige entre varias opciones dirigidas a lograr esencialmente el mismo resultado, y las probabilidades de que se use son mayores (5). Este tipo de análisis, sin embargo, se puede efectuar con regularidad solamente en algunos países. Por ende, el principal beneficio actual de esa disciplina radica en que promueve el reconocimiento de esta forma de pensar y facilita la toma de decisiones operativas sobre la factibilidad de una intervención y sus posibles consecuencias. A este tipo de ponderación a veces se le llama economía política.

Eso nos lleva de nuevo al punto de partida, es decir, a reconocer que las prioridades se ven enormemente influenciadas por consideraciones sociopolíticas, que son la cuarta fase dominante del proceso de adopción de decisiones. Al presentar evidencia objetiva de eficacia y efectividad y fomentar un mayor respeto por ellas entre las autoridades normativas, es posible que el proceso se lleve a cabo con el respaldo de más información.

\section{PARTICIPACIÓN, IDENTIFICACIÓN Y LIDERAZGO}

Al pasar de la evidencia al desempeño, los principales componentes del proceso son la partici- 
pación y la identificación con el problema y su solución. El otro elemento clave es el liderazgo, que se da de varias maneras: por delante o por detrás, saliendo del paso o simplemente despejando el camino. Existe, sin embargo, un elemento en común; para ser dirigente hay que tener seguidores. ;De nada vale que fijemos prioridades si nadie las observa! Aunque la participación en la fijación de prioridades y en la adopción de decisiones no garantiza el éxito, promoverá el trabajo en equipo, la identificación con el problema y su solución, y el compromiso de alcanzar determinadas metas y objetivos (6).

\section{DEFINICIÓN DEL PROBLEMA}

La primera dificultad cuando se pasa de la evidencia al desempeño es definir el problema. Esta tarea, más compleja de lo que parece a primera vista, depende del paradigma que empleemos. Los problemas y sus soluciones adquieren un aspecto diferente cuando es un individuo quien los examina y no una organización. Al examinar un problema, el economista se fijará en las funciones de producción; el epidemiólogo en la carga de morbilidad para la población; el fabricante en las posibles pérdidas y ganancias, y la comunidad en las necesidades mutuas de sus miembros.

No obstante, los problemas y sus soluciones también pueden definirse de manera integrada. No es necesario, por ejemplo, tratar a las enfermedades cardiovasculares, los accidentes cerebrovasculares, la insuficiencia renal crónica y la diabetes como si fueran entidades que se excluyen mutuamente. Desde el punto de vista de la prevención, tienen en común varios factores de riesgo que determinan su frecuencia y sus complicaciones, entre ellas una alimentación rica en grasas, la inactividad física y el consumo de tabaco. Asimismo, existen ciertos problemas relacionados con la política pública y la comunidad cuya resolución exige la participación de todos los interesados. En el nivel de la atención primaria de salud, la detección y el control de la hipertensión arterial son problemas que todos comparten y constituyen un reto para los servicios de prevención clínica. Es más probable que un método integrado de "salud para todos" lleve a más satisfacción y a una mejor atención de salud a un menor costo total. La más notable reducción de la incidencia de enfermedades cardiovasculares en el mundo se ha logrado en Finlandia en los últimos 20 años con un método integrado (7). Más adelante se describen las actividades del proyecto CARMEN en Chile (8). Sin embargo, las prioridades y decisiones dependen de quién decide y con qué paradigma. ¡La definición del problema conduce a su solución!

\section{PREGUNTAS FUNDAMENTALES SOBRE PLANIFICACIÓN}

Cuando se obtienen datos para respaldar la adopción de decisiones, surgen cuatro interrogantes relacionados con la planificación.

1) ¿Dónde estamos? Esta pregunta exige un análisis de la situación y una definición del problema (como se describió anteriormente). Si no nos ponemos de acuerdo sobre la naturaleza del problema, no podremos avanzar.

2) ¿Dónde queremos estar? Esta pregunta exige que haya metas y objetivos. Las Iniciativas de Cooperación para la Salud (Caribbean Cooperation in Health) en el Caribe y de Gente Sana 2010 (Healthy People 2010) en los Estados Unidos son ejemplos de experiencias en el campo de la fijación de metas.

Sin embargo, al mismo tiempo que abogamos por la fijación de metas de salud, debemos reconocer que esta es un arma de doble filo. Ciertos planes nacionales de salud tienen objetivos que leídos parecen muy admirables. Un ejemplo es el de "reducir en $50 \%$ la prevalencia de diabetes en un lapso de 10 años con métodos de promoción de la salud". Semejante meta es poco realista si aún no se ha establecido la base, si el programa tiene poca infraestructura instalada y si se está dando por sentado que todo lo que es posible en el plano teórico en verdad se llevará a la práctica, y todo ello en un momento en que se espera que la frecuencia de diabetes aumente casi otro tanto en el próximo decenio (9). En cuanto a las pandemias como la diabetes y el sida, lo que más razonablemente se puede esperar de una sólida programación de salud pública (en la mayoría de los contextos) es una desaceleración de la tasa de incidencia de la enfermedad. Las metas y los objetivos deben ser válidos en su aspecto epidemiológico y racionales en su aspecto administrativo; han de concebirse para el éxito, en vez de ser invitaciones al fracaso.

3) ¿Cómo llegaremos? Ahora debemos elaborar el plan de operaciones y los indicadores. Según Lembcke, "La mejor medida de la calidad no es cuán bien se presta un servicio médico ni con cuánta frecuencia, sino cuánto se acerca el resultado a los objetivos fundamentales de prolongar la vida, aliviar el sufrimiento, restituir la función y evitar la discapacidad" (10). Asimismo Donabedian indica que "Lograr y producir salud y satisfacción es el factor primordial de validación de la calidad de la atención" (11). Es probable que todos estemos de acuerdo con estas afirmaciones. Si bien es cierto que a los investigadores clínicos podría resultarles necesario cambiar su paradigma de los procesos a los resultados y que ello es factible en el caso de muchas enfermeda- 
des agudas, es preciso utilizar "el paradigma de los procesos" si hemos de promover un método basado en mediciones para establecer programas dirigidos a la atención de enfermedades crónicas.

En términos más explícitos, no es razonable pedir a los países en desarrollo que reestablezcan vínculos causales entre las actividades y sus resultados que ya han sido demostrados en otros contextos antes de lanzar iniciativas para el control de las enfermedades crónicas, sobre todo cuando esos resultados podrían tardar de 10 a 15 años en aparecer, o incluso más. Lo que necesitamos en la realidad es dar impulso a técnicas más novedosas para la evaluación de procesos, a sabiendas, por ejemplo, de que las personas que han recibido instrucción en materia de diabetes reducirán su concentración de $\mathrm{HbA} 1 \mathrm{c}, \mathrm{y}$ que ello a la vez reducirá la incidencia de complicaciones. Por lo tanto, la dificultad radica en instituir medidores de procesos sencillos y relativamente baratos que nos indiquen si estamos bien encauzados o no. Si volvemos a la afirmación de Peter Drucker, este es el punto en que podemos justificar un cambio de enfoque para centrarnos ya no en hacer lo adecuado, sino en hacer adecuadamente lo que es adecuado.

No interpreten mis palabras equivocadamente. La medición de los resultados es importante, pero al no realizarse cuando aún está en fase temprana la evolución de una enfermedad crónica, no es de utilidad inmediata para los administradores de programas. Necesitaremos disponer de indicadores de resultados cuando llegue el momento de la planificación final.

4) ¿Cómo sabremos que hemos llegado a ese punto? Algunos instrumentos de vigilancia bien elaborados, como el sistema de información que monitorea el desempeño del programa DIABCARE para personas con diabetes, aplican una combinación de medidores de procesos y de resultados (12).

\section{DISPONIBILIDAD Y CALIDAD DE LOS DATOS Y SUPOSICIONES SUBYACENTES}

Las preguntas ¿qué datos nos gustaría tener? y ¿qué datos tenemos? suelen generar dos respuestas diferentes: por lo general carecemos de los datos ideales y nos vemos obligados a trabajar con los datos que están a nuestro alcance. Eso significa que conviene reconocer sus limitaciones y suposiciones subyacentes, aun mientras luchamos por mejorar su disponibilidad y calidad.

¿Cuántos datos son suficientes? Desde el punto de vista operativo, lo que se necesita en realidad es disponer de datos esenciales mínimos, y ello varía según el fin deseado. Para establecer prioridades por lo general se necesitan relativamente pocos datos, siempre que sean razonablemente fiables (este es, en otras palabras, "el mundo del más o menos"). Sin embargo, para fines de la planificación y evaluación de programas se necesitan datos con un alto grado de especificidad y precisión. En este caso, es preciso contar con administradores que presten atención a los detalles según el alcance, que puede ser local, provincial, o nacional.

\section{FACTORES DECISIVOS PARA EL ÉXITO}

Otros factores decisivos para el éxito dentro del ámbito administrativo comprenden la disposición de los planificadores para "traducir" la evidencia en información que sirva de guía a las autoridades decisorias, y para formular objetivos e indicadores válidos y realistas. Los Centros para la Prevención y el Control de Enfermedades de los Estados Unidos realizan una buena labor en este sentido, como ilustra el "Programa de Transferencia sobre Diabetes". De importancia igualmente decisiva es la disposición de las instancias decisorias para consultar, buscar opiniones consensuales y tomar decisiones. A veces los países pobres aventajan a los otros en ese campo, ya que no pueden darse el lujo de hacer una elección costosa. Por último está la voluntad del gerente o administrador, cuya disposición para aceptar las decisiones, obrar de acuerdo con ellas y observar sus efectos es el factor más decisivo cuando se busca conseguir un cambio.

En todos los países, las necesidades de salud superan a los recursos disponibles. Por lo general esto se toma en un sentido presupuestario, pero naturalmente los recursos también están integrados por infraestructuras y tecnologías que suelen reflejan los paradigmas, prioridades, decisiones y acciones del pasado. Cualquier tentativa de efectuar un cambio mediante la fijación de prioridades también repercute en la cantidad y calidad de los recursos humanos. Aunque las fuentes de apoyo externo y la formación de alianzas nuevas son elementos importantes, en su mayor parte la movilización de recursos para hacer frente a esas necesidades debe ser interna: en principio, la obtención de buenos resultados en un campo determinado debe liberar los recursos para hacer frente a otras dificultades ("el dividendo de la salud"), pero eso no siempre sucede y debemos preguntarnos por qué.

¡Estoy resuelto; no me confundan con los hechos! Los propios ministros de salud deben inclinarse lo suficiente hacia el lado científico de la iniciativa para poder ponderar los hechos y estar alerta ante el posible uso de los datos de manera indebida. El simple hecho de haber sido emitida por una persona destacada no significa que una declaración sea ver- 
dadera. Podría ser un simple error que se fue propagando hasta ascender a ese nivel, o el resultado de la hábil defensa de intereses políticos. Cada vez es mayor la frecuencia con que los políticos se ven obligados a tomar decisiones sobre la base de conocimientos científicos. Como dijo la nueva Directora General de la OMS, médica salubrista y ex Primera Ministra de Noruega: “¿Por qué dejar la política ... que es la actividad de mayor relevancia dentro de una sociedad democrática ... en manos de una persona sin ningún conocimiento de la ciencia?" (13)

\section{CONCLUSIÓN}

La fijación de prioridades de salud es algo más que un ejercicio tecnocrático; es una mezcla de arte y ciencia y exige valor y liderazgo. En el mejor de los casos, equilibra los valores sociales con los mejores datos disponibles para incrementar la posibilidad de tomar decisiones acertadas. Las buenas decisiones, fundadas en un proceso racional, nos llevarán de la evidencia al desempeño.

\section{SYNOPSIS}

\section{From evidence to performance: setting priorities and making good decisions}

In today's world, with its respect for the medical sciences and demand for administrative accountability, health institutions are publicly responsible for their actions to an unprecedented degree. Making decisions and setting priorities on the basis of politics or unvalidated expert opinions is no longer acceptable. Instead, proven efficiency and effectiveness, as demonstrated by scientific evidence under continuous evaluation, are essential to decision-making. The need for applying rational criteria makes it necessary to explore a fundamental question: How can we promote critical reasoning within health services so as to adequately set priorities, make proper decisions, and launch effective interventions? In other words, how can we move from evidence to performance and why set priorities at all?

\section{REFERENCIAS}

1. Gribble JN, Preston SH, eds. The epidemiological transition: Policy and planning implications for developing countries. Washington, DC: National Academy Press; 1993.

2. Health Canada. Canada health action: Building on the legacy. Final report of the National Forum on Health. Ottawa: Health Canada; 1997.

3. Robles SC, White F, Peruga A. Tendencias de la mortalidad por cáncer de cuello de útero en las Américas. Bol Oficina Sanit Panam 1996;121:478-490.

4. Cochrane AL. Effectiveness and efficiency: Random reflections on health services. London: The Nuffield Provincial Hospitals Trust; 1972.

5. Drummond MF. Economic appraisal of health technology in the European commu- nity. New York: Oxford University Press; 1987.

6. Senge PM. The fifth discipline: The art and practice of the learning organization. New York: Doubleday Currency; 1990.

7. Puska P, Tuomilehto J, Nissinen A, Vartiainen E. The North Karelia Project: 20 year results and experiences. Helsinki: National Public Health Institute; 1995.

8. Peruga A. Conjunto de acciones para la reducción multifactorial de las enfermedades no transmisibles (CARMEN). En: Worldwide efforts to improve heart health. Atlanta: U.S. Centers for Disease Control and Prevention; 1997.

9. White F. Declaración de las Américas sobre la Diabetes: tema especial. IDF Bulletin (versión en español) 1997;42: 10-14.
10. Lembcke PA. Measuring quality of medical care through vital statistics based on hospital service areas. Am J Public Health 1952;42:276-286.

11. Donabedian A. Evaluating the quality of medical care. Milbank Mem Fund $Q$ 1966;44:166-203.

12. Krans HMJ, Porta M, Keen H. Diabetes care and research in Europe: the St. Vincent Declaration and Action Programme. Geneva: World Health Organization. (EUR/ICP/CLR 055/3).

13. Bruntland GH. Mensaje ante el Consejo Ejecutivo de la Organización Mundial de la Salud. Ginebra, Suiza, 19 a 28 de enero de 1998. Ginebra: OMS; 1998. 